\title{
Stochastic Exponential Stabilization for Markov Jump Neural Networks with Time-varying Delays via Adaptive Event-Triggered Impulsive Control
}

\author{
Xiaoman Liu, ${ }^{1}$ Haiyang Zhang $\mathbb{D}^{1,2}$ Tao $W u\left(\mathbb{D},{ }^{3}\right.$ and Jinlong Shu $\mathbb{D}^{4}$ \\ ${ }^{1}$ School of Mathematics and Computer Science, Yunnan Minzu University, Kunming 650500, China \\ ${ }^{2}$ School of Science, Nanjing University of Science and Technology, Nanjing 210094, China \\ ${ }^{3}$ Department of Mathematics, Southeast University, Nanjing 210096, China \\ ${ }^{4}$ School of Mathematics and Information Science, Shaanxi Normal University, Xi'an 710062, China
}

Correspondence should be addressed to Haiyang Zhang; haiya287@126.com

Received 10 February 2020; Revised 18 May 2020; Accepted 28 May 2020; Published 27 June 2020

Academic Editor: Yong Xu

Copyright (C) 2020 Xiaoman Liu et al. This is an open access article distributed under the Creative Commons Attribution License, which permits unrestricted use, distribution, and reproduction in any medium, provided the original work is properly cited.

\begin{abstract}
This paper focuses on the exponential stabilization problem for Markov jump neural networks with Time-varying Delays (TDs). Firstly, we provide a new Free-matrix-based Exponential-type Integral Inequality (FMEII) containing the information of attenuation exponent, which is helpful to reduce the conservativeness of stability criteria. To further save control cost, we introduce a sample-based Adaptive Event-triggered Impulsive Control (AEIC) scheme, in which the trigger threshold is adaptively varied with the sampled state. By fully considering the information about sampled state, TDs, and Markov jump parameters, a suitable Lyapunov-Krasovskii functional is constructed. With the virtue of FMEII and AEIC scheme, some novel stabilization criteria are presented in the form of linear matrix inequalities. At last, two numerical examples are given to show the validity of the obtained results.
\end{abstract}

\section{Introduction}

Recent decades have witnessed the fast development of neural networks since its wide applications in many practical fields, such as pattern recognition [1], smart antenna arrays [2], and circuit design [3]. In the implementation of such applications, both Time-varying Delays (TDs) caused by the inherent communication time among neurons or the finite switching speed of amplifier [4-8] and random abrupt arisen from the external environment sudden change or the information latching [9-11] are inevitably encountered, which often lead to some undesirable dynamic behaviors, such as chaotic, oscillation, and even unstable [12-14]. Markov Jump Neural Networks (MJNNs) with Time-varying Delays (TDs), as a special kind of hybrid system, has a powerful ability in describing those complicated behaviors. Stability is a precondition for the normal operation of systems, and sometimes fast convergence of the networks is essential for real-time computation. As it is well known, the exponential convergence rate is generally used to determine the speed of neural computations [15]. Thus, it is of great theoretical and practical importance to study the exponential stability for MJNNs with TDs, and many fruit results have been reported in the literature [16-18].

In particular, under an assumption that the proportional delay is unbounded time-varying, some global stochastic exponential stability conditions for MJNNs with proportional TDs are derived in [16]. By considering a more general uncertain transition rates, Liu et al. [17] investigated the stochastic exponential stability for neutral-type impulsive MJNNs with mixed TDs. With the aid of random analysis method and Lyapunov functional techniques, Zhang et al. [18] presented some exponential stability results for MJNNs with TDs and general disturbance. It is worth mentioning that the Exponential-type Integral Quadratic Terms (EIQTs), i.e., $-\int_{\delta_{1}}^{\delta_{2}} e^{\alpha\left(s-\delta_{2}\right)} \varphi^{T}(s) \Xi \varphi(s) \mathrm{d} s$, which play crucial role in 
deriving less conservative stability criteria [16-21]. In most of the existing literature, the upper bound of EIQTs are usually estimated by using some nonexponential-type integral inequalities, such as Free-matrix-based Integral Inequality (FMII) [19], Wirtinger's integral inequality $[16-18,20]$, and other kind of integral inequality [21]. However, there exists a common shortcoming in such kinds of inequalities, that is, the information of attenuation exponent is not considered fully, which brings some conservativeness to some extent. Recently, some Exponential-type Integral Inequalities (EIIs) have been proposed and successfully applied to the exponential stability analysis for time-delay systems $[22,23]$. Nerveless, it cannot find more tighter upper bound for the EIQTs, since the parameter matrices in EIIs are not adjustable. Consequently, it should be possible to reduce the conservativeness of results based on the ideas of FMII [19] and EIIs [23], which is the first motivation of our paper.

On the contrary, Event-triggered Impulsive Control (EIC), as a powerful tool, plays an important role in many different science and engineering fields. Especially, in the field of artificial neural networks, the study for stabilization is more complicated due to the state-dependent nonlinear switching behaviors of neural networks. Compared with the common impulsive control, EIC scheme may avoid some undesirable network behaviors, such as some "unnecessary" data are sent frequently, and the network resources are excessively used to some extent, especially in the case that the networks resources are limited [24]. Therefore, EIC scheme has attracted much more attention in many different fields, multiagent systems [25], nonlinear systems [26, 27], neural networks [28, 29], and so on [30, 31]. It should be pointed out that there exist some disadvantages in the above EIC schemes in [25-31]. For example, the supervising time of sensors is in the mean of continuous time, which implies that the sensors are required to monitor the system state all the time $[25,30,31]$, but it is not necessary because the worst scenario rarely happens. Besides, the triggering threshold is assumed to be a constant [27-29], which reduces the flexibility of EIC schemes and limits the applications ability of them. Furthermore, to overcome the above disadvantages, a sample-based AEIC scheme is introduced in this paper, where the trigger threshold is adaptively varied with the sampled state. So far as it is known to the authors, few literature studies have devoted to the exponential stabilization problem for MJNNs with TDs via a samplebased AEIC scheme, which is another motivation of our paper.

Motivated by the aforementioned discussions, the main contributions of our paper are summarized as follows. (1) Inspired by the ideas of FMII [19] and EIIs [23], a new FMEII is proposed to derive more tighter bound of the EIQTs in this paper, which is more general and flexible than the existing EIIs. (2) In order to overcome the shortcomings in existing EIC schemes, a sample-based AEIC scheme is introduced, where the triggering threshold is governed by a well designed adaptive rule corresponded to the sampled state. (3) The FMEII and AEIC scheme are successfully applied to investigate the exponential stabilization issue for MJNNs with TDs, and some novel stabilization results are derived in terms of linear matrices inequalities.

Notations: let $\mathbb{N}$ denote the set of positive integers, $\mathbb{R}$ the set of real numbers, $\mathbb{R}^{n}$ the $n$-dimensional real space equipped with the Euclidean norm $\|\cdot\|, \mathbb{R}^{m \times n}$ the set of all $m \times n$ real matrices, and $\mathscr{R}_{+}^{n}$ and $\mathscr{R}^{n}$ the set of symmetric positive definite and symmetric matrices of $\mathbb{R}^{n \times n}$, respectively. Denote $\langle f, g\rangle_{w}=\int_{a}^{b} f(s) g(s) w(s) \mathrm{d} s$ for any integrable functions $f(\cdot), g(\cdot), w(\cdot)$ in $[a, b]$. The symbol "*” in a block matrix signifies the symmetric terms, $\operatorname{col}\{\cdots\}$ and $\operatorname{diag}\{\ldots\}$ express a column vector and a diagonal matrix, respectively. For any matrix $X \in \mathbb{R}^{n \times n}, \mathbb{H}\{X\}$ means that $X+$ $X^{T}$ and $\lambda_{\max }(X)$ and $\lambda_{\min }(X)$ stand for the maximum and minimum eigenvalue of $X$, respectively. The zero and identity matrices with appropriate dimensions are described by 0 and $I$, respectively. The symbol $\bar{e}_{i}=(0, \ldots$, $0, \underbrace{I}_{i}, 0, \ldots, 0,),(i=1, \ldots, 14)$.

\section{Description of Problem and Preliminaries}

Let $\{r(t), t \geq 0\}$ be a continuous-time Markov process taking values in a finite state space $\mathfrak{N}=(1,2, \ldots, \mathcal{N})$. The evolution of $\{r(t), t \geq 0\}$ is governed by the following transition probability:

$$
\operatorname{Pr}\{r(t+\Delta)=j \mid r(t)=i\}= \begin{cases}\pi_{i j} \Delta+o(\Delta), & i \neq j, \\ 1+\pi_{i i} \Delta+o(\Delta), & i=j,\end{cases}
$$

where $\Delta \geq 0, \lim _{\Delta \longrightarrow 0} o(\Delta) / \Delta=0 ; \pi_{i j} \geq 0$ for $i \neq j \in \mathfrak{N}$ is the transition rate from mode $i$ at time $t$ to mode $j$ at time $t+\Delta$, and $\pi_{i i}=-\sum_{j=1, j \neq i}^{\mathcal{N}} \pi_{i j}$.

Consider the following impulsive MJNNs with TDs:

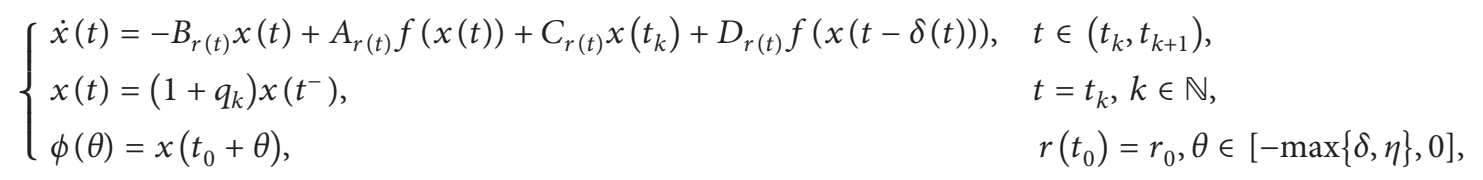

where $x(t)=\operatorname{col}\left\{x_{1}(t), \ldots, x_{n}(t)\right\}$ is the state; $f(x(t))=$ $\operatorname{col}\left\{f_{1}\left(x_{1}(t)\right), \ldots, f_{n}\left(x_{n}(t)\right)\right\}$ is the neuron activation $\lambda_{l}^{-} \leq \frac{f_{l}\left(x_{1}\right)-f_{l}\left(x_{2}\right)}{x_{1}-x_{2}} \leq \lambda_{l}^{+}, \quad f_{l}(0)=0, l \in \mathbb{N}, \forall x_{1} \neq x_{2} \in \mathbb{R}$, 
where $\lambda_{l}^{-}$and $\lambda_{l}^{+}$are scalars which can be positive, negative, and zero; $\phi(\theta)$ is the initial condition; and $\delta(t)$ is a timevarying delay and satisfies

$$
\begin{aligned}
0 & \leq \delta_{1} \leq \delta(t) \leq \delta_{2}, \\
\dot{\delta}(t) & \leq \mu,
\end{aligned}
$$

where $\delta_{1}, \delta_{2}$, and $\mu$ are known constants; $B_{r(t)}$ is a positive diagonal matrix and $A_{r(t)}$ and $D_{r(t)}$ are connection weighted matrices; $C_{r(t)}$ is control gain matrix to be determine, $q_{k}$ is impulsive intensity, $t_{k}$ is impulsive instant, $x\left(t_{k}\right)=x\left(t_{k}^{+}\right)=$ $\lim _{\varepsilon \longrightarrow 0^{+}} x\left(t_{k}+\varepsilon\right), x\left(t_{k}^{-}\right)=\lim _{\varepsilon \longrightarrow 0^{-}} x\left(t_{k}+\varepsilon\right)$, and $r_{0} \in \mathfrak{N}$ is the initial mode as $t=t_{0}$.

To further mitigate unnecessary waste of network resources, a sample-based AEIC scheme is introduced in this paper. Assume that the system's state is periodically sampled and the sampling sequence is depicted by the set $\Pi_{s}=\{0, h, 2 h, \ldots, k h\}$ with $k \in \mathbb{N}$, where $h$ is constant sampling period, and the event-triggered sequence is described by the set $\Pi_{e}=\left\{0, b_{1} h, b_{2} h, \ldots, b_{k} h\right\} \subseteq \Pi_{s}$ with $b_{k} \in \mathbb{N}$. Suppose the event-triggered instants to be the impulsive instants, i.e., $t_{k}=b_{k} h$, then the next impulsive instant $t_{k+1}=t_{k}+l_{m} h$, where

$$
\begin{aligned}
l_{m}= & \min \left\{l \mid e^{T}\left(t_{k}+l h\right) \Omega e\left(t_{k}+l h\right)>\sigma\left(t_{k}+l h\right) x^{T}\right. \\
& \left.\cdot\left(t_{k}+l h\right) \Omega x\left(t_{k}+l h\right)\right\}, \quad l \in \mathbb{N},
\end{aligned}
$$

and $\Omega \in \mathscr{R}_{+}^{n}$ is an unknown weighted matrix, $e\left(t_{k}+l h\right)=$ $x\left(t_{k}+l h\right)-x\left(t_{k}\right)$ expresses the error between the two states at the latest trigger instant and the current sampling one, and trigger threshold $\sigma\left(t_{k}+l h\right) \in[0,1)$ is governed by the following adaptive rule:

$$
\sigma\left(t_{k}+(l+1) h\right)=\max \left\{\sigma\left(t_{k}+l h\right) \Delta\left(t_{k}+l h\right), \sigma_{m}\right\},
$$

where

$\Delta\left(t_{k}+l h\right)=1-\left(2 \Delta_{1} / \pi\right) \arctan \left[\Delta_{2}\left(\left\|x\left(t_{k}+l h\right)\right\|-\left\|x\left(t_{k}\right)\right\|\right)\right]$, $\arctan (\cdot)$ is the invert tangent function, $\Delta_{1}, \Delta_{2}>0$ are given constants to adjust the output of $\arctan (\cdot), \sigma_{m}$ is the given lower bound of $\sigma\left(t_{k}+l h\right)$, and $\sigma(0)=\sigma_{m}$.

Remark 1. Note that the function $\arctan (\cdot)$ in adaptive rule (6) has the lower and upper bounds, i.e., $\operatorname{actan}(\cdot) \in[-\pi / 2$, $\pi / 2]$, and this property can be used in this paper combining with adjustable parameters $\Delta_{1}$ and $\Delta_{2}$ to adaptively adjust the trigger threshold $\sigma\left(t_{k}+l h\right)$. Specifically, when the norm of the current sampling state is larger than the one of the last triggered state, that is, $\left\|x\left(t_{k}+l h\right)\right\|>\left\|x\left(t_{k}\right)\right\|$, then we can obtain that $\Delta\left(t_{k}+l h\right) \in(0,1)$ and $\sigma\left(t_{k}+(l+1) h\right)$ $<\sigma\left(t_{k}+l h\right)$, which means that, in this case, the adaptive rule
(6) uses smaller $\sigma\left(t_{k}+(l+1) h\right)$ to reduce the error between $\left\|x\left(t_{k}+(l+1) h\right)\right\|$ and $\left\|x\left(t_{k}\right)\right\|$ at next sampling instant, and if the trigger condition is satisfied, the trigger threshold will be updated as $\sigma\left(t_{k+1}\right)$ at the same time. The inverse case is similar with the above discussions, and it is omitted here. Besides, the existence of parameters $\Delta_{1}$ and $\Delta_{2}$ can ensure that the threshold $\sigma\left(t_{k}+l h\right)$ are always belonging to $[0,1)$ as the parameters $\Delta_{1}$ and $\Delta_{2}$ are adjustable.

Remark 2. It is familiar to use the following EIC scheme:

$$
t_{k+1}=\inf \left\{t \mid t>t_{k},\left\|x(t)-x\left(t_{k}\right)\right\| \geq \sigma\left\|x\left(t_{k}\right)\right\|\right\},
$$

which has been widely used in recent years $[28,30,31]$. However, there are some undesirable traits in scheme (7). For example, (i) the EIC scheme (7) does not need to be carried out if the system state decreases very fast in a very short time, even though it satisfies the trigger condition. (ii) It requires the sensors to monitor the system state all the time. Clearly, methods that require continuous monitoring of the systems could waste network resources much more because the worst errors that can happen, such as external disturbance, network congestion, or packet dropout are rarely evident. Hence, the sample-based AEIC scheme (5) saves more communication cost than the scheme (7), because it only needs supervision at sampling instants. Furthermore, the Zeno behavior can be naturally avoided by using the sample-based AEIC scheme (5), since the impulse interval satisfies that $\inf \left\{t_{k+1}-t_{k}\right\} \geq h>0$ for all $t \in\left[t_{k}, t_{k+1}\right), k \in \mathbb{Z}_{+}$. (iii) Moreover, under the pregiven constant threshold $\sigma$, the EIC scheme (7) cannot deal with the case that the trajectory of the system oscillates along with a certain line and does not converge in a relatively large interval. In these senses, the trigger threshold $\sigma\left(t_{k}+l h\right)$ in scheme (5) is more reasonable, flexible, and can be adaptively adjusted by according to the adaptive rule (6).

For the sake of introducing the sample-based AEIC scheme to determine whether the current sampled-data should be transmitted, an effective way is to consider the sampled-data error at every sampling instant. Decompose the impulsive interval $\left[t_{k}, t_{k+1}\right)$ into the following subinterval $\left[t_{k}, t_{k+1}\right)=\cup_{l=0}^{l_{m}-1} \square_{k}(l)$, where $\mathbb{q}_{k}(l)=\left[t_{k}+l h, t_{k}+(l+1) h\right)$. Define a function:

$$
\eta(t)=t-\left(t_{k}+l h\right), \quad t \in \mathbb{\mathbb { V }}_{k}(l) .
$$

Note that $\eta(t)$ is a linear piecewise function and satisfies $0 \leq \eta(t) \leq \eta, \dot{\eta}(t)=1$, and $\forall t \in \mathbb{\mathbb { V }}_{k}(l)$. For simplifying some notations, denote $B_{r(t)}=B_{i}, A_{r(t)}=A_{i}, D_{r(t)}=D_{i}$, and $C_{r(t)}=C_{i}$ when $r(t)=i$. Then, combining with (5) and (8), system (2) can be rewritten as

$$
\begin{cases}\dot{x}(t)=-B_{i} x(t)+A_{i} f(x(t))+D_{i} f(x(t-\delta(t)))+C_{i} x(t-\eta(t))-C_{i} e(t-\eta(t)), & t \in\left(t_{k}, t_{k+1}\right), \\ x(t)=\left(1+q_{k}\right) x\left(t^{-}\right), & t=t_{k}, k \in \mathbb{N}, \\ \phi(\theta)=x\left(t_{0}+\theta\right), & r(0)=r_{0}, \theta \in[-\max \{\delta, \eta\}, 0] .\end{cases}
$$


The following definition and lemmas need to be recalled, which play a key role to demonstrate our main results.

Definition 1 (see [9]). System (9) is said to be stochastically exponentially stable in the mean square sense with convergence rate $\alpha>0$, if there exist scalar $M>0$ for $\forall t \geq t_{0}$ such that

$$
\mathbb{E}\left\{\|x(t)\|^{2}\right\} \leq M e^{-\alpha\left(t-t_{0}\right)} \mathbb{E}\left\{\sup _{\theta \in[-\max \{\delta, \eta\}, 0]}\left\{\|\phi(\theta)\|^{2},\|\dot{\phi}(\theta)\|^{2}\right\}\right\} .
$$

Lemma 1 (see [22]). For a given matrix $\Xi \in \mathscr{R}_{+}^{n}$, scalars $a<b, \quad \alpha>0$, and $a$ differentiable vector function $\xi(s):[a, b] \longrightarrow \mathbb{R}^{n}$, the following inequality holds:

$$
\begin{aligned}
& -\int_{a}^{b} e^{\alpha(s-b)} \xi^{T}(s) \Xi \xi(s) \mathrm{d} s \leq-\frac{\alpha}{e^{\alpha(b-a)}-1}\left(\int_{a}^{b} \xi(s) \mathrm{d} s\right)^{T} \\
& \Xi\left(\int_{a}^{b} \xi(s) \mathrm{d} s\right)
\end{aligned}
$$

Remark 3. Clearly, when $\varphi(s)=\dot{x}(s)$, one can obtain from (11) that

$$
\begin{gathered}
-\int_{a}^{b} e^{\alpha(s-b)} \dot{x}^{T}(s) \Xi \dot{x}(s) \mathrm{d} s \leq-\frac{\alpha}{e^{\alpha(b-a)}-1}[x(b)-x(a)]^{T} \\
\Xi[x(b)-x(a)] .
\end{gathered}
$$

Furthermore, when $\alpha \longrightarrow 0$, EII (11) will reduce to the well-known Jensen's integral inequality in [32]. Besides, it has been shown in [22] that a more tighter bound for EIQT can be derived by using EII (11). Very recently, some more accurate EIIs have been proposed in [23]; however, the FMEII that can enhance the flexibility of EII to some extent has not been found in the existing literature so far. Therefore, inspired by the ideas in $[19,23]$, we attempt to establish a simple kind of FMEII to further estimate the bound of EIQTs in this paper.

Lemma 2. For given matrices $\Xi \in \mathscr{R}_{+}^{n}, N \in \mathbb{R}^{m n \times n}$, any vector $\zeta \in \mathbb{R}^{m n}, m \in \mathbb{N}$, scalars $a<b$ and a differentiable vector function $\varphi(s):[a, b] \longrightarrow \mathbb{R}^{n}$, and there exist scalar auxiliary function $w(s) \geq 0$ with $s \in[a, b]$ such that the following inequality holds:

$$
-\int_{a}^{b} \varphi^{T}(s) \Xi \varphi(s) w(s) d s \leq\langle 1,1\rangle_{w} \zeta^{T} N \Xi^{-1} N^{T} \zeta+2 \zeta^{T} N\langle 1, \varphi\rangle_{w} .
$$

Proof. Note the fact that, for given matrices $\Xi \in \mathscr{R}_{+}^{n}$, $N \in \mathbb{R}^{m n \times n}$, any vector $\zeta \in \mathbb{R}^{m n}, m \in \mathbb{N}$, scalars $a<b$, and scalar auxiliary function $w(s) \geq 0$ with $s \in[a, b]$, one has

$$
-2 \varphi^{T}(s) N^{T} \zeta w(s) \leq \zeta^{T} N \Xi^{-1} N^{T} \eta w(s)+\varphi^{T}(s) \Xi \varphi(s) w(s) .
$$

Integrating both sides of (14) from $a$ to $b$, we have

$$
\begin{aligned}
-\int_{a}^{b} \varphi^{T}(s) \Xi \varphi(s) w(s) d s \leq & \int_{a}^{b} \zeta^{T} N \Xi^{-1} N^{T} \zeta w(s) d s \\
& +2 \int_{a}^{b} \zeta^{T} N \varphi(s) w(s) d s .
\end{aligned}
$$

Then, inequality (13) can be obtained after expanding the right side of inequality (15).

Remark 4. Clearly, when $\varphi(s)=w^{-1}(s) \xi(s), \quad w(s)=$ $e^{-\alpha(s-b)}, N=\Xi, \zeta=-\left(\gamma_{0} / \alpha\right) \int_{a}^{b} \xi(s) \mathrm{d} s$, and $\gamma_{0}=e^{\alpha(b-a)}-1$, inequality (13) will reduce to inequality (11), which implies that Lemma 1 can be regarded as a special case of Lemma 2. On the contrary, when $\varphi(s)=w^{-1}(s) \dot{x}(s)$ and $w(s)=$ $e^{-\alpha(s-b)}$, inequality (13) can be rewritten as follows:

$$
\begin{aligned}
-\int_{a}^{b} e^{\alpha(s-b)} \dot{x}^{T}(s) \Xi \dot{x}(s) \mathrm{d} s \leq & \frac{\gamma_{0}}{\alpha} \zeta^{T} N \Xi^{-1} N^{T} \zeta \\
& +2 \zeta^{T} N[x(b)-x(a)] .
\end{aligned}
$$

Due to the existence of vector $\zeta$ and free matrix $N$, the flexibility of (13) can be enhanced to some degree. For example, if $\zeta=\operatorname{col}\{x(b), \dot{x}(b), x(a), \dot{x}(a)\}$ and $N=\operatorname{col}\left\{N_{1}\right.$, $\left.N_{2}, N_{3}, N_{4}\right\}$, one can obtain that

$$
-\int_{a}^{b} e^{\alpha(s-b)} \dot{x}^{T}(s) \Xi \dot{x}(s) \mathrm{d} s \leq \frac{\gamma_{0}}{\alpha} \zeta^{T} N \Xi^{-1} N^{T} \zeta+\zeta^{T} \widehat{N} \zeta,
$$

where

$$
\widehat{N}=\left[\begin{array}{cccc}
\mathbb{H}\left\{N_{1}\right\} & N_{2}^{T} & -N_{1}+N_{3}^{T} & N_{4}^{T} \\
* & 0 & -N_{2} & 0 \\
* & * & \mathbb{L}\left\{N_{3}\right\} & -N_{4}^{T} \\
* & * & * & 0
\end{array}\right] .
$$

It is easy to see that some connections between vectors $x(b)$ and $x(a)$ and other augment vectors are established, which may facilitate the estimation for the bounds of EIQTs.

\section{Main Results}

In this section, our purpose is to establish new stochastic exponential stabilization conditions for systems (9) via the AEIC scheme (5). Before presenting the main results, the following vectors are defined for convenience: 


$$
\begin{aligned}
\xi(t)= & \operatorname{col}\left\{x(t), f(x(t)), x(t-\delta(t)), f(x(t-\delta(t))), x\left(t-\delta_{1}\right), x\left(t-\delta_{2}\right), x(t-\eta(t)), x(t-\eta), e(t-\eta(t))\right. \\
& \left.\int_{t-\delta(t)}^{t-\delta_{1}} \frac{x(s)}{\delta(t)-\delta_{1}} \mathrm{~d} s \int_{t-\delta_{2}}^{t-\delta(t)} \frac{x(s)}{\delta_{2}-\delta(t)} \mathrm{d} s, \int_{t-\eta(t)}^{t} \frac{x(s)}{\eta(t)} \mathrm{d} s, \int_{t-\eta}^{t-\eta(t)} \frac{x(s)}{\eta-\eta(t)} \mathrm{d} s, \dot{x}(t)\right\} \\
\zeta_{21}= & \operatorname{col}\left\{\bar{e}_{5}-\bar{e}_{3}, \bar{e}_{5}+\bar{e}_{3}-2 \bar{e}_{10}\right\} \\
\zeta_{22}= & \operatorname{col}\left\{\bar{e}_{3}-\bar{e}_{6}, \bar{e}_{3}+\bar{e}_{6}-2 \bar{e}_{11}\right\} \\
\zeta_{31}= & \operatorname{col}\left\{\bar{e}_{1}-\bar{e}_{7}, \bar{e}_{1}+\bar{e}_{7}-2 \bar{e}_{12}\right\} \\
\zeta_{32}= & \operatorname{col}\left\{\bar{e}_{7}-\bar{e}_{8}, \bar{e}_{7}+\bar{e}_{8}-2 \bar{e}_{13}\right\}
\end{aligned}
$$

Theorem 1. For given positive scalars $\delta_{1}, \delta_{2}, \mu, \eta, \alpha$, and $\sigma_{m}$, system (9) is said to be stochastically exponentially stable in the mean square sense, if there exist matrices $P_{i}, S_{i}, Q_{1}, Q_{2}$, $Q_{3}, \quad Q_{4}, \quad Q_{5}, \quad Q_{6}, \quad R_{1}, \quad R_{2}, \quad R_{3} \in \mathscr{R}_{+}^{n}, X_{1} \in \mathbb{R}^{6 n \times n}, X_{2}$, $X_{3} \in \mathbb{R}^{2 n \times 2 n}, N_{i}, K_{i} \in \mathbb{R}^{n \times n}$ and diagonal matrix $M_{1}$, $M_{2} \in \mathscr{R}_{+}^{n}$ such that

$$
\begin{gathered}
\left(1+q_{k}\right)^{2} \lambda_{\max }\left(P_{i}+S_{i}\right)-\lambda_{\min }\left(P_{i}\right)>0, \quad k \in \mathbb{N}, \\
\inf \left\{t_{k+1}-t_{k}\right\}=\beta>\frac{\ln q}{\alpha}, \quad k \in \mathbb{N},
\end{gathered}
$$

$$
\begin{gathered}
\left(\begin{array}{cc}
\mathscr{R}_{2} & X_{2} \\
* & \mathscr{R}_{2}
\end{array}\right)>0,\left(\begin{array}{cc}
\mathscr{R}_{3} & X_{3} \\
* & \mathscr{R}_{3}
\end{array}\right)>0, \\
\left(\begin{array}{cc}
\Phi_{1 i}+\Phi_{2 i}+\Phi_{3 i}+\Phi_{4 i}+\Phi_{5 i} & \zeta_{1}^{T} X_{1} \\
* & -\frac{\alpha}{\gamma_{0}} R_{1}
\end{array}\right)<0,
\end{gathered}
$$

where $q=\left(1+q_{k}\right)^{2} \lambda_{\max }\left(P_{i}+S_{i}\right) / \lambda_{\min }\left(P_{i}\right), \quad \gamma_{0}=e^{\alpha \delta_{1}}-1$, and

$$
\begin{aligned}
& \Phi_{1 i}=\llbracket\left\{\bar{e}_{1}^{T} P_{i} \bar{e}_{14}\right\}+\bar{e}_{1}^{T}\left(\alpha P_{i}+\Pi\left(P_{j}\right)\right) \bar{e}_{1}+\left(\bar{e}_{7}-\bar{e}_{9}\right)^{T}\left(\alpha S_{i}+\Pi\left(S_{j}\right)\right)\left(\bar{e}_{7}-\bar{e}_{9}\right), \\
& \Phi_{2 i}=\bar{e}_{1}^{T}\left(Q_{1}+Q_{3}+Q_{4}+Q_{5}+Q_{6}\right) \bar{e}_{1}+\bar{e}_{2}^{T} Q_{2} \bar{e}_{2}-e^{-\alpha \delta_{2}}(1-\mu) \bar{e}_{3}^{T} Q_{1} \bar{e}_{3} \\
& -e^{-\alpha \delta_{2}}(1-\mu) \bar{e}_{4}^{T} Q_{2} \bar{e}_{4}-e^{-\alpha \delta_{1}} \bar{e}_{5}^{T} Q_{3} \bar{e}_{5}-e^{-\alpha \delta_{2}} \bar{e}_{6}^{T} Q_{4} \bar{e}_{6}-e^{-\alpha \eta} \bar{e}_{8}^{T} Q_{6} \bar{e}_{8}, \\
& \Phi_{3 i}=\bar{e}_{14}^{T}\left(\delta_{1} R_{1}+\left(\delta_{2}-\delta_{1}\right)^{2} R_{2}+\eta^{2} R_{3}\right) \bar{e}_{14}+\nVdash\left\{\zeta_{1}^{T} X_{1}\left(\bar{e}_{1}-\bar{e}_{5}\right)\right\} \\
& -e^{-\alpha \delta_{2}}\left(\begin{array}{c}
\zeta_{21} \\
\zeta_{22}
\end{array}\right)^{T}\left(\begin{array}{cc}
\mathscr{R}_{2} & X_{2} \\
* & \mathscr{R}_{2}
\end{array}\right)\left(\begin{array}{c}
\zeta_{21} \\
\zeta_{22}
\end{array}\right)-e^{-\alpha \eta}\left(\begin{array}{c}
\zeta_{31} \\
\zeta_{32}
\end{array}\right)^{T}\left(\begin{array}{cc}
\mathscr{R}_{3} & X_{3} \\
* & \mathscr{R}_{3}
\end{array}\right)\left(\begin{array}{l}
\zeta_{31} \\
\zeta_{32}
\end{array}\right), \\
& \Phi_{4 i}=\sigma_{m} \bar{e}_{7}^{T} \Omega \bar{e}_{7}-\bar{e}_{9}^{T} \Omega \bar{e}_{9}+\mathbb{U}\left\{\left(\bar{e}_{14}+\bar{e}_{1}\right)^{T} K_{i}\left(\bar{e}_{7}-\bar{e}_{9}\right)\right\}+\mathbb{U}\left\{\left(\bar{e}_{14}+\bar{e}_{1}\right)^{T} N_{i}\left(-\bar{e}_{14}-B_{i} \bar{e}_{1}+A_{i} \bar{e}_{2}+D_{i} \bar{e}_{4}\right)\right\}, \\
& \Phi_{5 i}=-\mathbb{H}\left\{\left(\bar{e}_{2}-\Lambda_{1} \bar{e}_{1}\right)^{T} M_{1}\left(\bar{e}_{2}-\Lambda_{2} \bar{e}_{1}\right)+\left(\bar{e}_{4}-\Lambda_{1} \bar{e}_{3}\right)^{T} M_{2}\left(\bar{e}_{4}-\Lambda_{2} \bar{e}_{3}\right)\right\}, \\
& \Pi\left(P_{j}\right)=\sum_{j=1}^{\mathcal{N}} \pi_{i j} P_{j}, \Pi\left(S_{j}\right)=\sum_{j=1}^{\mathcal{N}} \pi_{i j} S_{j}, \\
& \mathscr{R}_{l}=\operatorname{diag}\left\{R_{l}, 3 R_{l}\right\}, \quad(l=2,3) .
\end{aligned}
$$

In addition, the control gain is designed by $C_{i}=N_{i}^{-1} K_{i}, i \in \mathfrak{N}$.
Proof. Consider the following stochastic Lyapunov-Krasovskii Functional (LKF): 


$$
\begin{aligned}
V(x(t), r(t))= & V_{1}(x(t), r(t))+V_{2}(x(t), r(t)) \\
& +V_{3}(x(t), r(t)),
\end{aligned}
$$

$$
\begin{aligned}
V_{1}(x(t), r(t))= & x^{T}(t) P\left(r_{t}\right) x(t)+x^{T}\left(t_{k}\right) S(r)_{t} x\left(t_{k}\right) \\
V_{2}(x(t), r(t))= & \int_{t-\delta(t)}^{t} e^{\alpha(s-t)}\left[x^{T}(s) Q_{1} x(s)+f^{T}(x(s)) Q_{2} f(x(s))\right] \mathrm{d} s \\
& +\int_{t-\delta_{1}}^{t} e^{\alpha(s-t)} x^{T}(s) Q_{3} x(s) \mathrm{d} s+\int_{t-\delta_{2}}^{t} e^{\alpha(s-t)} x^{T}(s) Q_{4} x(s) \mathrm{d} s \\
& +\int_{t-\eta(t)}^{t} e^{\alpha(s-t)} x^{T}(s) Q_{5} x(s) \mathrm{d} s+\int_{t-\eta}^{t} e^{\alpha(s-t)} x^{T}(s) Q_{6} x(s) \mathrm{d} s, \\
V_{3}(x(t), r(t))= & \int_{-\delta_{1}}^{0} \int_{t+u}^{t} e^{\alpha(s-t)} \dot{x}^{T}(s) R_{1} \dot{x}(s) \mathrm{d} s \mathrm{~d} u \\
& +\left(\delta_{2}-\delta_{1}\right) \int_{-\delta_{2}}^{-\delta_{1}} \int_{t+u}^{t} e^{\alpha(s-t)} \dot{x}^{T}(s) R_{2} \dot{x}(s) \mathrm{d} s \mathrm{~d} u \\
& +\eta \int_{-\eta}^{0} \int_{t+u}^{t} e^{\alpha(s-t)} \dot{x}^{T}(s) R_{3} \dot{x}(s) \mathrm{d} s \mathrm{~d} u .
\end{aligned}
$$

Let $\mathscr{L}$ be the week infinitesimal operator acting on LKF (25), i.e.,

$$
\mathscr{L} V(x(t), r(t))=\lim _{\Delta \longrightarrow 0^{+}} \frac{\mathbb{E}\{V(x(t+\Delta), r(t+\Delta)=j) \mid x(t), r(t)=i\}-V(x(t), i)}{\Delta},
$$

then along with the solution of systems (9), we have

$$
\begin{aligned}
\mathscr{L} V_{1}(x(t), i)= & 2 \dot{x}^{T}(t) P_{i} x(t)+x^{T}(t) \Pi\left(P_{j}\right) x(t)+x^{T}\left(t_{k}\right) \Pi\left(s_{j}\right) x\left(t_{k}\right) \\
= & \xi^{T}(t) \Phi_{1 i} \xi(t)-\alpha V_{1}(x(t), i), \\
\mathscr{L} V_{2}(x(t), i) \leq & x^{T}(t)\left(Q_{1}+Q_{3}+Q_{4}+Q_{5}+Q_{6}\right) x(t)+f^{T}(x(t)) Q_{2} f(x(t)) \\
& -(1-\mu) e^{-\alpha \delta} x^{T}(t-\delta(t)) Q_{1} x(t-\delta(t)) \\
& -(1-\mu) e^{-\alpha \delta} f^{T}\left(x(t-\delta(t)) Q_{2} f(x(t-\delta(t)))\right) \\
& -e^{-\alpha \delta_{1}} x^{T}\left(t-\delta_{1}\right) Q_{3} x\left(t-\delta_{1}\right)-e^{-\alpha \delta_{2}} x^{T}\left(t-\delta_{2}\right) Q_{4} x\left(t-\delta_{2}\right) \\
& -e^{-\alpha \eta} x^{T}(t-\eta) Q_{6} x(t-\eta)-\alpha V_{2}(x(t), i) \\
= & \xi^{T}(t) \Phi_{2 i} \xi(t)-\alpha V_{2}(x(t), i), \\
\mathscr{L} V_{3}(x(t), i) \leq & \dot{x}^{T}(t)\left[\delta_{1} R_{1}+\left(\delta_{2}-\delta_{1}\right)^{2} R_{2}+\eta^{2} R_{3}\right] \dot{x}(t) \\
& -\int_{t-\delta_{1}}^{t} e^{\alpha(s-t)} \dot{x}^{T}(s) R_{1} \dot{x}(s) \mathrm{d} s-\eta e^{-\alpha \eta} \int_{t-\eta}^{t} \dot{x}^{T}(s) R_{3} \dot{x}(s) \mathrm{d} s \\
& -\left(\delta_{2}-\delta_{1}\right) e^{-\alpha \delta_{2}} \int_{t-\delta_{2}}^{t-\delta_{1}} \dot{x}^{T}(s) R_{2} \dot{x}(s) \mathrm{d} s-\alpha V_{3}(x(t), i) .
\end{aligned}
$$


For vector $\zeta=\operatorname{col}\left\{x(t), x(t-\delta(t)), x\left(t-\delta_{1}\right), x\left(t-\delta_{2}\right)\right.$, $x(t-\eta(t)), x(t-\eta)\}$ and matrix $X_{1} \in \mathbb{R}^{6 n \times n}$, it follows from Lemma 2 that

$$
\begin{aligned}
& -\int_{t-\delta_{1}}^{t} e^{\alpha(s-t)} \dot{x}^{T}(s) R_{1} \dot{x}(s) \mathrm{d} s \\
& \quad \leq \xi^{T}(t)\left\{\frac{\gamma_{0}}{\alpha} \zeta_{1}^{T} X_{1} R_{1}^{-1} X_{1}^{T} \zeta_{1}+2 \zeta_{1}^{T} X_{1}\left[\bar{e}_{1}-\bar{e}_{5}\right]\right\} \xi(t),
\end{aligned}
$$

where $\zeta_{1}=\operatorname{col}\left\{\bar{e}_{1}, \bar{e}_{3}, \bar{e}_{5}, \bar{e}_{6}, \bar{e}_{7}, \bar{e}_{8}\right\}$. Meanwhile, by utilizing Lemmas 1 and 3 in [33], one can obtain that

$$
\begin{aligned}
& -\left(\delta_{2}-\delta_{1}\right) e^{-\alpha \delta_{2}} \int_{t-\delta_{2}}^{t-\delta_{1}} \dot{x}^{T}(s) R_{2} \dot{x}(s) \mathrm{d} s \\
& \leq \xi^{T}(t)\left\{-e^{-\alpha \delta_{2}}\left(\begin{array}{c}
\zeta_{21} \\
\zeta_{22}
\end{array}\right)^{T}\left(\begin{array}{cc}
\mathscr{R}_{2} & X_{2} \\
* & \mathscr{R}_{2}
\end{array}\right)\left(\begin{array}{c}
\zeta_{21} \\
\zeta_{22}
\end{array}\right)\right\} \xi(t), \\
& -\eta e^{-\alpha \eta} \int_{t-\eta}^{t} \dot{x}^{T}(s) R_{3} \dot{x}(s) \mathrm{d} s \\
& \leq \xi^{T}(t)\left\{-e^{-\alpha \eta}\left(\begin{array}{c}
\zeta_{31} \\
\zeta_{32}
\end{array}\right)^{T}\left(\begin{array}{cc}
\mathscr{R}_{3} & X_{3} \\
* & \mathscr{R}_{3}
\end{array}\right)\left(\begin{array}{c}
\zeta_{31} \\
\zeta_{32}
\end{array}\right)\right\} \xi(t) .
\end{aligned}
$$

Combining (30)-(33), we have

$$
\begin{gathered}
\mathscr{L} V_{3}(x(t), i) \leq \xi^{T}(t)\left\{\frac{\gamma_{0}}{\alpha} \zeta_{1}^{T} X_{1} R_{1}^{-1} X_{1}^{T} \zeta_{1}+\Phi_{3 i}\right\} \\
\xi(t)-\alpha V_{3}(x(t), i) .
\end{gathered}
$$

In addition, when the current data does not need to be sent out, it is easy to know from the AEIC scheme (5) that $0<\sigma_{m} x^{T}(t-\eta(t)) \Omega x(t-\eta(t))-e^{T}(t-\eta(t)) \Omega e(t-\eta(t))$

$$
=\xi^{T}(t)\left\{\sigma_{m} \bar{e}_{7}^{T} \Omega \bar{e}_{7}-\bar{e}_{9}^{T} \Omega \bar{e}_{9}\right\} \xi(t) .
$$

Furthermore, from (9), for any matrices $N_{i}, K_{i} \in \mathbb{R}^{n \times n}$, we have

$$
\begin{aligned}
0= & 2[\dot{x}(t)+x(t)]^{T} N_{i}\left[-B_{i} x(t)+A_{i} f(x(t))\right. \\
& \left.+D_{i} f(x(t-d(t)))\right] \\
& +2[\dot{x}(t)+x(t)]^{T} K_{i}[x((t-\eta(t))-e(t-\eta(t))] \\
= & \xi^{T}(t)\left\{\mathbb{M}\left\{\left(\bar{e}_{14}+\bar{e}_{1}\right)^{T} K_{i}\left(\bar{e}_{7}-\bar{e}_{9}\right)\right\}\right. \\
& \left.+\mathbb{U}\left\{\left(\bar{e}_{14}+\bar{e}_{1}\right)^{T} N_{i}\left(-\bar{e}_{14}-B_{i} \bar{e}_{1}+A_{i} \bar{e}_{2}+D_{i} \bar{e}_{4}\right)\right\}\right\} \xi(t) .
\end{aligned}
$$

According to (3), there exist diagonal matrices $M_{1}, M_{2} \in \mathscr{R}_{+}^{n}$ such that

$$
\begin{aligned}
0 \leq & -2\left[f(x(t))-\Lambda_{1} x(t)\right]^{T} M_{1}\left[f(x(t))-\Lambda_{2} x(t)\right] \\
= & \xi^{T}(t)\left\{-\mathbb{E}\left\{\left(\bar{e}_{2}-\Lambda_{1} \bar{e}_{1}\right)^{T} M_{1}\left(\bar{e}_{2}-\Lambda_{2} \bar{e}_{1}\right)\right\}\right\} \xi(t), \\
0 \leq & -2\left[f(x(t-d(t)))-\Lambda_{1} x(t-d(t))\right]^{T} \\
& \cdot M_{2}\left[f(x(t-d(t)))-\Lambda_{2} x(t-d(t))\right] \\
= & \xi^{T}(t)\left\{-\mathbb{Q}\left\{\left(\bar{e}_{4}-\Lambda_{1} \bar{e}_{3}\right)^{T} M_{2}\left(\bar{e}_{4}-\Lambda_{2} \bar{e}_{3}\right)\right\}\right\} \xi(t),
\end{aligned}
$$

where $\Lambda_{1}=\operatorname{diag}\left\{\lambda_{1}^{-}, \ldots, \lambda_{n}^{-}\right\}$and $\Lambda_{2}=\operatorname{diag}\left\{\lambda_{1}^{+}, \ldots, \lambda_{n}^{+}\right\}$.

Therefore, combining with (28)-(38) and (22), one can obtain

$$
\mathscr{L} V(t) \leq-\alpha V(t)
$$

By utilizing Dynkin formula [10] and Grownwall inequality, one has

$$
\mathbb{E}\{V(t)\} \leq e^{-\alpha\left(t-t_{k}\right)} \mathbb{E}\left\{V\left(t_{k}\right)\right\}, \forall t \in\left[t_{k}, t_{k+1}\right) .
$$

From (9), (19), and (23), it follows that

$$
\begin{aligned}
\mathbb{E}\left\{V\left(t_{k}\right)-V\left(t_{k}^{-}\right)\right\} & \leq \mathbb{E}\left\{x\left(t_{k}^{-}\right)\left(1+q_{k}\right)^{2}\left(P_{i}+S_{i}\right) x\left(t_{k}^{-}\right)\right\}-\mathbb{E}\left\{x\left(t_{k}^{-}\right) P_{i} x\left(t_{k}^{-}\right)\right\} \\
& \leq\left[\left(1+q_{k}\right)^{2} \lambda_{\max }\left(P_{i}+S_{i}\right)-\lambda_{\min }\left(P_{i}\right)\right] \mathbb{E}\left\{\left\|x\left(t_{k}^{-}\right)\right\|^{2}\right\} \\
& \leq(q-1) \mathbb{E}\left\{x\left(t_{k}^{-}\right) P_{i} x\left(t_{k}^{-}\right)\right\} \leq(q-1) V\left(t_{k}^{-}\right),
\end{aligned}
$$

which implies that $V\left(t_{k}\right) \leq q V\left(t_{k}^{-}\right)$.

Hence, combining with (40) and (21), we have

$$
\begin{aligned}
\mathbb{E}\{V(t)\} & \leq q^{k} e^{-\alpha\left(t-t_{0}\right)} \mathbb{E}\left\{V\left(t_{0}\right)\right\} \\
& \leq e^{-(\alpha-(\ln q / \beta))\left(t-t_{0}\right)} \mathbb{E}\left\{V\left(t_{0}\right)\right\}, \forall t \geq t_{0} .
\end{aligned}
$$

It is easy to know from (25) that

$$
\begin{aligned}
& \mathbb{E}\{V(t)\} \geq \lambda_{\min }\left(P_{i}\right) \mathbb{E}\left\{\|x(t)\|^{2}\right\}, \\
& \mathbb{E}\left\{V\left(t_{0}\right)\right\} \leq\left(\gamma_{1}+\gamma_{2}+\gamma_{3}\right) \sup \left\{\|\phi(\theta)\|^{2},\|\dot{\phi}(\theta)\|^{2}\right\},
\end{aligned}
$$




$$
\begin{aligned}
\gamma_{1}= & \lambda_{\max }\left(P_{i}+S_{i}\right), \Lambda=\operatorname{diag}\left\{\lambda_{1}, \ldots, \lambda_{n}\right\}, \lambda_{\tilde{\nu}}=\max \left\{\left|\lambda_{\bar{\nu}}\right|,\left|\lambda_{\nu}^{+}\right|\right\}, \quad \widetilde{\nu}=1,2, \ldots, n, \\
\gamma_{2}= & \frac{1-e^{-\alpha \delta_{2}}}{\alpha}\left[\lambda_{\max }\left(Q_{1}\right)+\lambda_{\max }\left(Q_{2}\right)\|\Lambda\|^{2}+\lambda_{\max }\left(Q_{4}\right)\right] \\
& +\frac{1-e^{-\alpha \delta_{1}}}{\alpha} \lambda_{\max }\left(Q_{3}\right)+\frac{1-e^{-\alpha \eta}}{\alpha}\left[\lambda_{\max }\left(Q_{5}\right)+\lambda_{\max }\left(Q_{6}\right)\right], \\
\gamma_{3}= & \frac{\left(e^{-\alpha \delta_{1}}+\alpha \delta_{1}-1\right)}{\alpha^{2}} \lambda_{\max }\left(R_{1}\right)+\eta \frac{\left(e^{-\alpha \eta}+\alpha \eta-1\right)}{\alpha^{2}} \lambda_{\max }\left(R_{3}\right) \\
& +\left(\delta_{2}-\delta_{1}\right) \frac{e^{-\alpha \delta_{2}}-e^{-\alpha \delta_{1}}+\alpha\left(\delta_{2}-\delta_{1}\right)}{\alpha^{2}} \lambda_{\max }\left(R_{2}\right) .
\end{aligned}
$$

From (42)-(44), one has

$$
\mathbb{E}\left\{\|x(t)\|^{2}\right\} \leq M e^{-\gamma\left(t-t_{0}\right)} \mathbb{E}\left\{\sup \left\{\|\phi(\theta)\|^{2},\|\dot{\phi}(\theta)\|^{2}\right\}\right\},
$$

where $M=\left(\gamma_{1}+\gamma_{2}+\gamma_{3}\right) / \lambda_{\min }\left(P_{i}\right)$ and $\gamma=\alpha-(\ln q / \beta)$.

Therefore, according to Definition 2, system (9) is said to be stochastically exponentially stable in the mean square sense with the convergence rate $\gamma>0$. In addition, the control gain is designed by $C_{i}=N_{i}^{-1} K_{i}, i \in \mathfrak{N}$. This completes the proof.

Remark 5. It should be pointed out that the constructed LKF $V(x(t), i)$ plays a key role in deriving the stochastic exponential stabilization result. Specifically, the impulsive signal related to Markov jump parameters is considered in
$V_{1}(x(t), i)$, and the information about the lower bound and upper bound of time-varying delay is taken into account in $V_{2}(x(t), i), V_{3}(x(t), i)$, which is more general than others given in [16-18] and is helpful to obtain less conservative stability criterion. Besides, in the proof of Theorem 1, we utilize a new FMEII to estimate the bound of EIQT, which was shown more tighter than the ones based on Jensen's integral inequality [32] and EII in [22]. Meanwhile, the reciprocally convex approach is employed to consider the relationship between the time-varying delay and its interval, which is effective to reduce the conservatism.

In what follows, as a special case, when Markov jump parameters are not considered, system (9) will be reduced to the following equation:

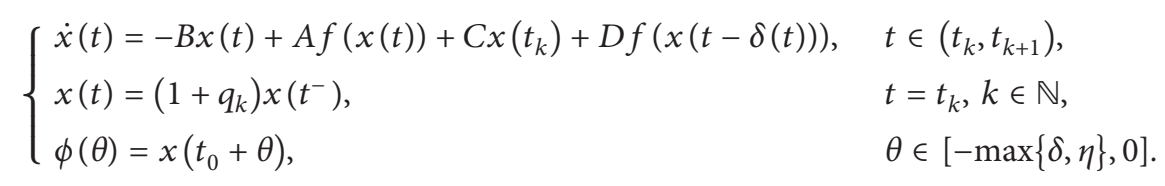

Based on Theorem 1, the following criterion can be readily derived.

Theorem 2. For given positive scalars $\delta_{1}, \delta_{2}, \mu, \eta, \alpha$, and $\sigma_{m}$, system (47) is said to be exponentially stable, if there exist matrices $P, S, Q_{1}, Q_{2}, Q_{3}, Q_{4}, Q_{5}, Q_{6}, R_{1}, R_{2}, R_{3} \in \mathscr{R}_{+}^{n}$, $X_{1} \in \mathbb{R}^{6 n \times n}, X_{2}, X_{3} \in \mathbb{R}^{2 n \times 2 n}, N, K \in \mathbb{R}^{n \times n}$ and diagonal matrix $M_{1}, M_{2} \in \mathscr{R}_{+}^{n}$ such that

$$
\begin{gathered}
\left(1+q_{k}\right)^{2} \lambda_{\max }(P+S)-\lambda_{\min }(P)>0, \quad k \in \mathbb{N}, \\
\inf \left\{t_{k+1}-t_{k}\right\}=\beta>\frac{\ln q}{\alpha}, \quad k \in \mathbb{N}, \\
\left(\begin{array}{cc}
\mathscr{R}_{2} & X_{2} \\
* & \mathscr{R}_{2}
\end{array}\right)>0,\left(\begin{array}{cc}
\mathscr{R}_{3} & X_{3} \\
* & \mathscr{R}_{3}
\end{array}\right)>0, \\
\left(\begin{array}{cc}
\widehat{\Phi}_{1}+\widehat{\Phi}_{2}+\widehat{\Phi}_{3}+\widehat{\Phi}_{4}+\widehat{\Phi}_{5} & \zeta_{1}^{T} X_{1} \\
* & -\frac{\alpha}{\gamma_{0}} R_{1}
\end{array}\right)<0,
\end{gathered}
$$


where $q=\left(1+q_{k}\right)^{2} \lambda_{\max }(P+S) / \lambda_{\min }(P), \gamma_{0}=e^{\alpha \delta_{1}}-1$, and

$$
\begin{aligned}
& \widehat{\Phi}_{1}=\mathbb{H}\left\{\bar{e}_{1}^{T} P \bar{e}_{14}\right\}+\alpha \bar{e}_{1}^{T} P \bar{e}_{1}+\alpha\left(\bar{e}_{7}-\bar{e}_{9}\right)^{T} S\left(\bar{e}_{7}-\bar{e}_{9}\right), \\
& \widehat{\Phi}_{2}=\bar{e}_{1}^{T}\left(Q_{1}+Q_{3}+Q_{4}+Q_{5}+Q_{6}\right) \bar{e}_{1}+\bar{e}_{2}^{T} Q_{2} \bar{e}_{2}-e^{-\alpha \delta_{2}}(1-\mu) \bar{e}_{3}^{T} Q_{1} \bar{e}_{3} \\
& -e^{-\alpha \delta_{2}}(1-\mu) \bar{e}_{4}^{T} Q_{2} \bar{e}_{4}-e^{-\alpha \delta_{1}} \bar{e}_{5}^{T} Q_{3} \bar{e}_{5}-e^{-\alpha \delta_{2}} \bar{e}_{6}^{T} Q_{4} \bar{e}_{6}-e^{-\alpha \eta} \bar{e}_{8}^{T} Q_{6} \bar{e}_{8}, \\
& \widehat{\Phi}_{3}=\bar{e}_{14}^{T}\left(\delta_{1} R_{1}+\left(\delta_{2}-\delta_{1}\right)^{2} R_{2}+\eta^{2} R_{3}\right) \bar{e}_{14}+\mathbb{Q}\left\{\left\{\zeta_{1}^{T} X_{1}\left(\bar{e}_{1}-\bar{e}_{5}\right)\right\}\right. \\
& -e^{-\alpha \delta_{2}}\left(\begin{array}{c}
\zeta_{21} \\
\zeta_{22}
\end{array}\right)^{T}\left(\begin{array}{cc}
\mathscr{R}_{2} & X_{2} \\
* & \mathscr{R}_{2}
\end{array}\right)\left(\begin{array}{c}
\zeta_{21} \\
\zeta_{22}
\end{array}\right)-e^{-\alpha \eta}\left(\begin{array}{l}
\zeta_{31} \\
\zeta_{32}
\end{array}\right)^{T}\left(\begin{array}{cc}
\mathscr{R}_{3} & X_{3} \\
* & \mathscr{R}_{3}
\end{array}\right)\left(\begin{array}{c}
\zeta_{31} \\
\zeta_{32}
\end{array}\right), \\
& \widehat{\Phi}_{4}=\sigma_{m} \bar{e}_{7}^{T} \Omega \bar{e}_{7}-\bar{e}_{9}^{T} \Omega \bar{e}_{9}+\mathbb{Q}\left\{\left(\bar{e}_{14}+\bar{e}_{1}\right)^{T} K\left(\bar{e}_{7}-\bar{e}_{9}\right)\right\} \\
& +\mathbb{Q}\left\{\left(\bar{e}_{14}+\bar{e}_{1}\right)^{T} N\left(-\bar{e}_{14}-B \bar{e}_{1}+A \bar{e}_{2}+D \bar{e}_{4}\right)\right\}, \\
& \widehat{\Phi}_{5}=-\mathbb{M}\left\{\left(\bar{e}_{2}-\Lambda_{1} \bar{e}_{1}\right)^{T} M_{1}\left(\bar{e}_{2}-\Lambda_{2} \bar{e}_{1}\right)+\left(\bar{e}_{4}-\Lambda_{1} \bar{e}_{3}\right)^{T} M_{2}\left(\bar{e}_{4}-\Lambda_{2} \bar{e}_{3}\right)\right\} .
\end{aligned}
$$

In addition, the control gain is designed by $C=N^{-1} K$.

\section{Numerical Examples}

In this section, we aim to demonstrate the feasibility and validity of the obtained results in this paper by two numerical examples.

Example 1. Consider system (9) with the following parameters [34-36]:

$$
\begin{aligned}
B_{1} & =\left[\begin{array}{ll}
1 & 0 \\
0 & 1
\end{array}\right], \\
A_{1} & =\left[\begin{array}{cc}
2 & -0.1 \\
-5 & 3
\end{array}\right], \\
D_{1} & =\left[\begin{array}{ll}
-1.5 & -0.1 \\
-0.2 & -2.5
\end{array}\right], \\
B_{2} & =\left[\begin{array}{cc}
0.8 & 0 \\
0 & 1
\end{array}\right], \\
A_{2} & =\left[\begin{array}{cc}
2 & -0.11 \\
-5 & 3.2
\end{array}\right], \\
D_{2} & =\left[\begin{array}{cc}
-1.6 & -0.1 \\
-0.18 & -2.4
\end{array}\right], \\
\left(\pi_{i j}\right) & =\left[\begin{array}{cc}
-3 & 3 \\
5 & -5
\end{array}\right], \\
\Lambda_{1} & =\operatorname{diag}\{0,0\}, \\
\Lambda_{2} & =\operatorname{diag}\{0.5,0.5\}, \\
\delta_{1} & =0.1, \\
\delta_{2} & =0.3 \\
\mu & =0.2, \\
\alpha & =0.1, \\
\sigma_{m} & =0.05, \\
\eta & =0.1 .
\end{aligned}
$$

Let the neuron activation function $f_{i}(x)=0.5(|x+1|-|x-1|)$, the time-varying delay $\delta(t)=0.2+0.2 \sin (t)$, the initial value $x(0)=[-0.2 ; 0.3]$, and the parameters $\Delta_{1}=1, \Delta_{2}=50$ in adaptive rule (6); Figures 1-4 are presented to show the feasibility and validity 
of the obtained result in this paper. Specifically, Figures 1 and 2 stand for the state response of system (9) with parameters (50) and (51) under no control and the welldesigned AEIC scheme (5), respectively. Clearly, AEIC scheme (5) is effective. Furthermore, it is not hard to see from Figure 3 that the frequency of impulsive control is reduced to large extent, which means that more network resources are saved by using the AEIC scheme. Moreover, compared with Figure 4, one can find that the triggering threshold adjusted adaptively by adaptive rule (6) is varying with the evolution of the system state, i.e., the threshold gradually decrease when the norm of the system state goes up, which enhances the flexibility of the AEIC scheme to some extent.

On the contrary, if the adaptive threshold $\sigma\left(t_{k}+l h\right)$ is a constant, that is, $\Delta_{2}=0$, then the AEIC scheme (5) will reduce to a EIC scheme (7). Without loss of generality, take the threshold in EIC scheme as $\sigma_{m}$, and under the same parameters with above mentioned, Figures 5 and 6 are given to show the results of ECI control scheme. It is clear from Figures 2 and 5 that both AEIC and EIC scheme can stabilise the underlying system (9), while comparing Figures 3 and 6 , and the control frequency of AEIC scheme is 27/50 and the control frequency of EIC scheme is $46 / 50$, where 50 is the number of sampling data. Clearly, AEIC scheme is better to decrease the frequency of impulsive control than the EIC scheme. Thus, it is concluded that the control cost can be saved more effectively by the AEIC scheme than the EIC scheme.

Example 2. Consider system (47) with the following parameters [34-36]:

$$
\begin{aligned}
A & =\left[\begin{array}{cc}
0.9+\frac{\pi}{4} & 19 \\
0.11 & 0.9+\frac{\pi}{4}
\end{array}\right], \\
D & =\left[\begin{array}{cc}
-1.2 \frac{\sqrt{2} \pi}{4} & 0.3 \\
0.2 & -1.2 \frac{\sqrt{2} \pi}{4}
\end{array}\right], \\
B & =\operatorname{diag}\{1,0.9\}, \\
\Lambda_{1} & =\operatorname{diag}\{0,0\}, \\
\Lambda_{2} & =\operatorname{diag}\{0.4,0.8\}, \\
\delta_{1} & =0.1, \\
\delta_{2} & =0.4, \\
\mu & =0.2, \\
\alpha & =0.2, \\
\sigma_{m} & =0.05, \\
\eta & =0.1 .
\end{aligned}
$$



Figure 1: Curve of $x(t)$ for Example 1 without any control.

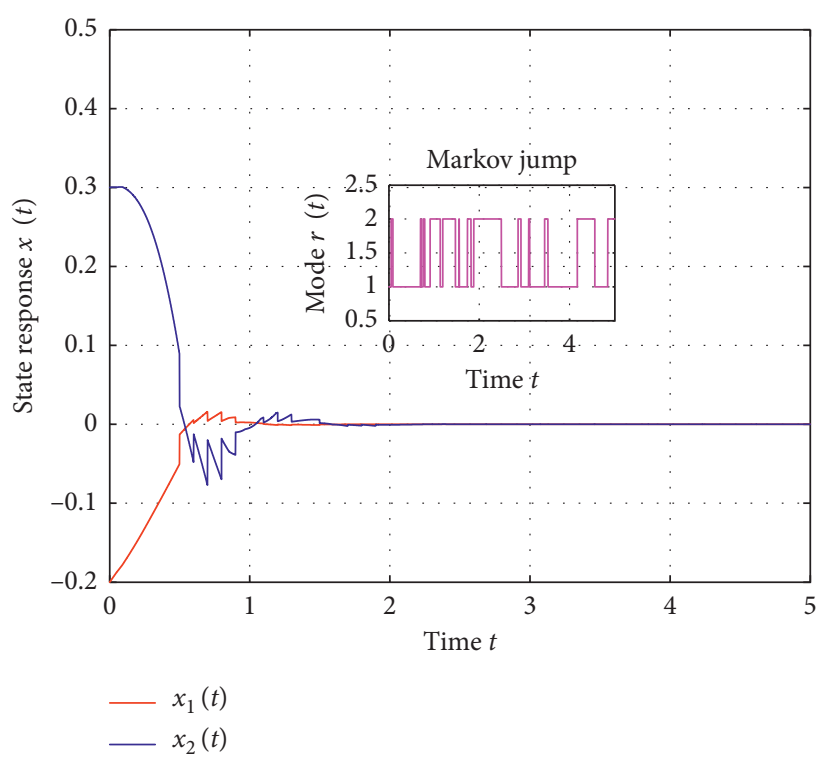

Figure 2: Curve of $x(t)$ for Example 1 under AEIC.

Under the above parameters, applying Theorem 2 and using the MATLAB LMI toolbox, one can derive that the impulsive intensity $q_{k}=-0.8884$ and the control gains matrix $C=\left[\begin{array}{ll}-2.5040 & -6.1983 \\ -0.1509 & -1.6798\end{array}\right]$. Meanwhile, the weight matrix $\Omega$ in the AEIC scheme and other unknown matrices in Theorem 2 can be obtained: 


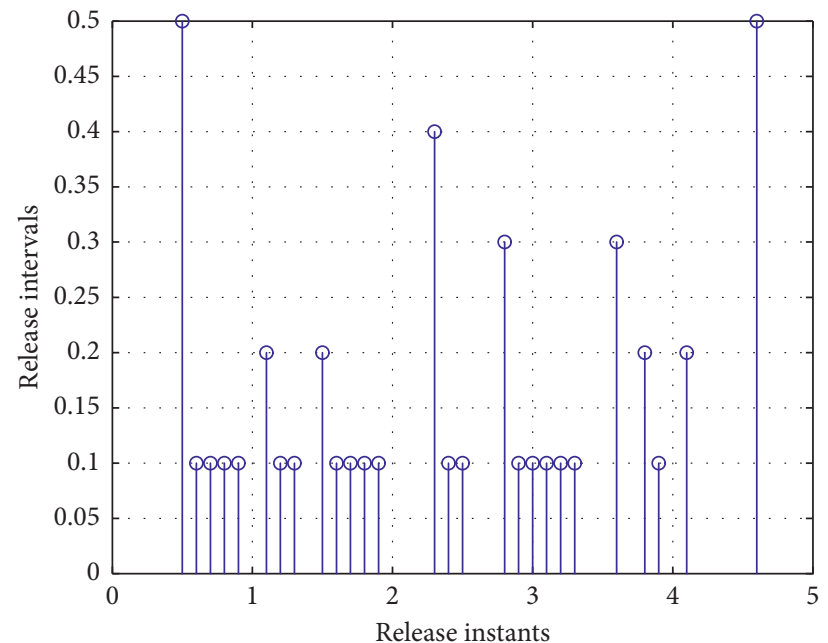

Figure 3: Release instants and intervals for Example 1 under AEIC.

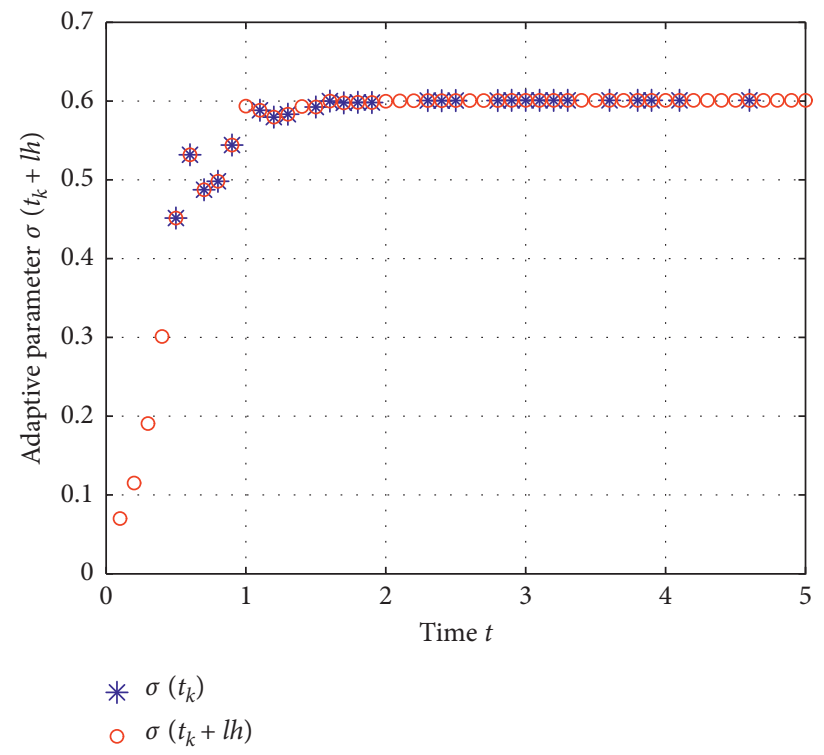

Figure 4: Adaptive parameters of AEIC scheme for Example 1.

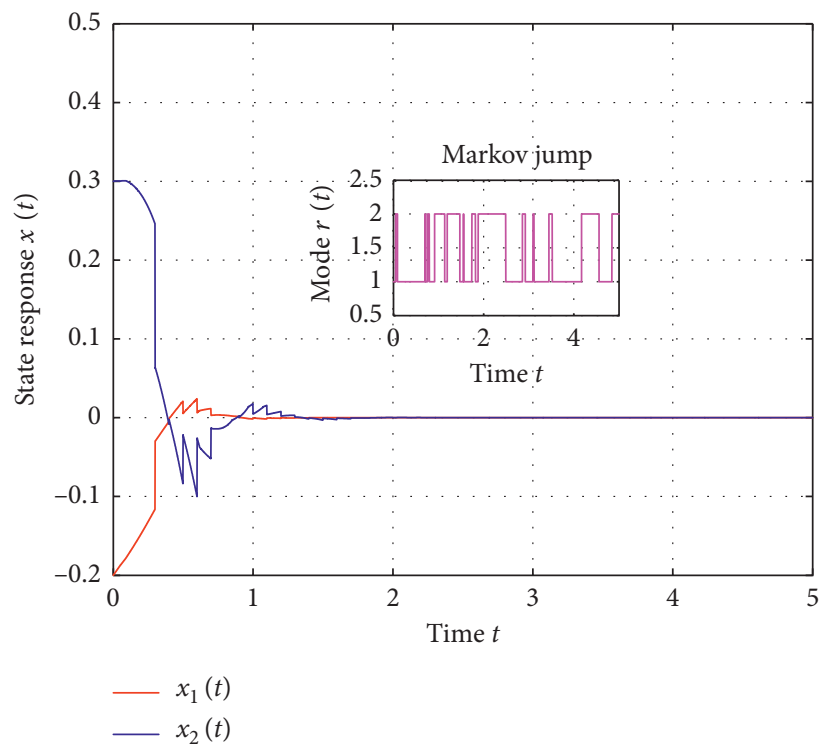

Figure 5: Curve of $x(t)$ for Example 1 under EIC. 


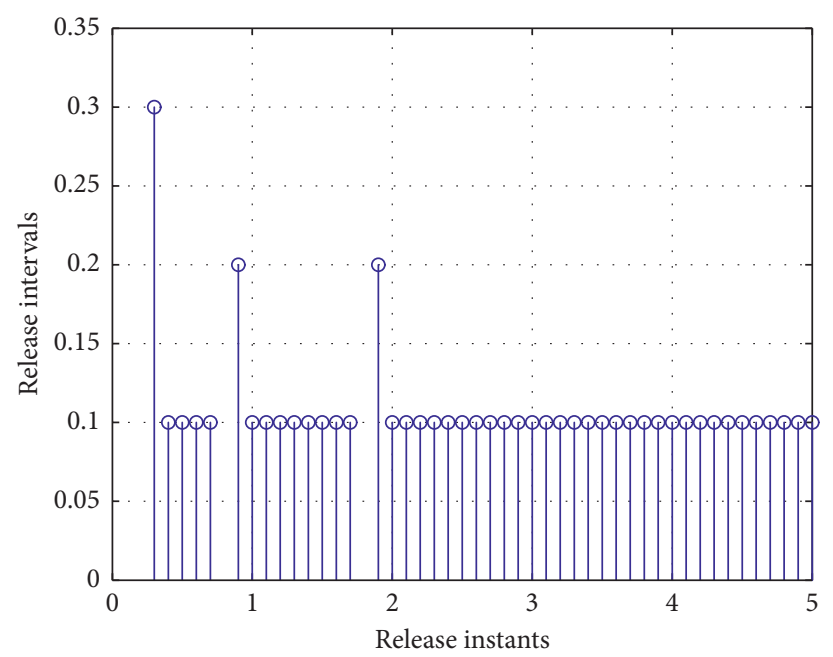

FIGURE 6: Release instants and intervals for Example 1 under EIC.

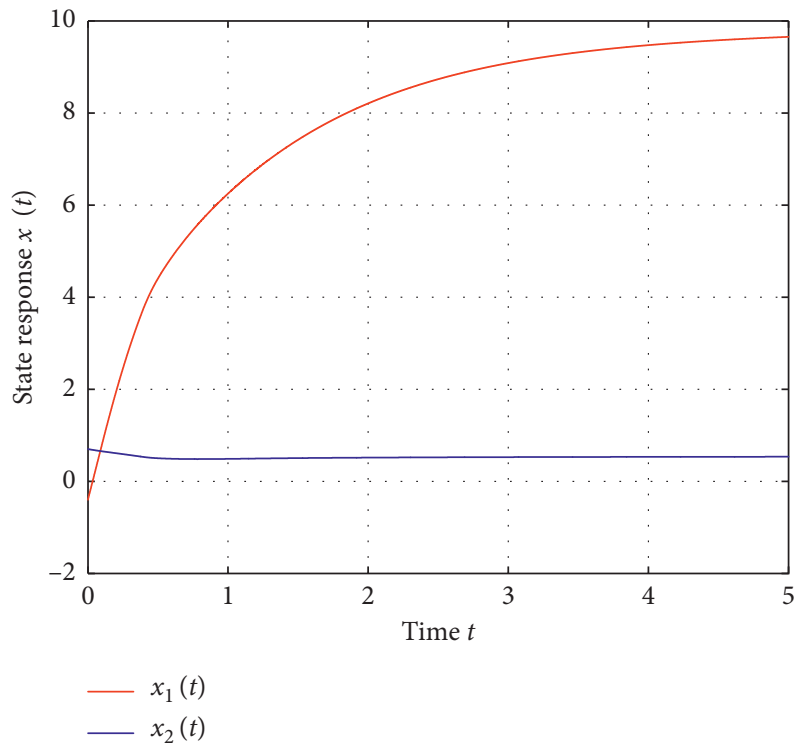

Figure 7: Curve of $x(t)$ without control for Example 2.

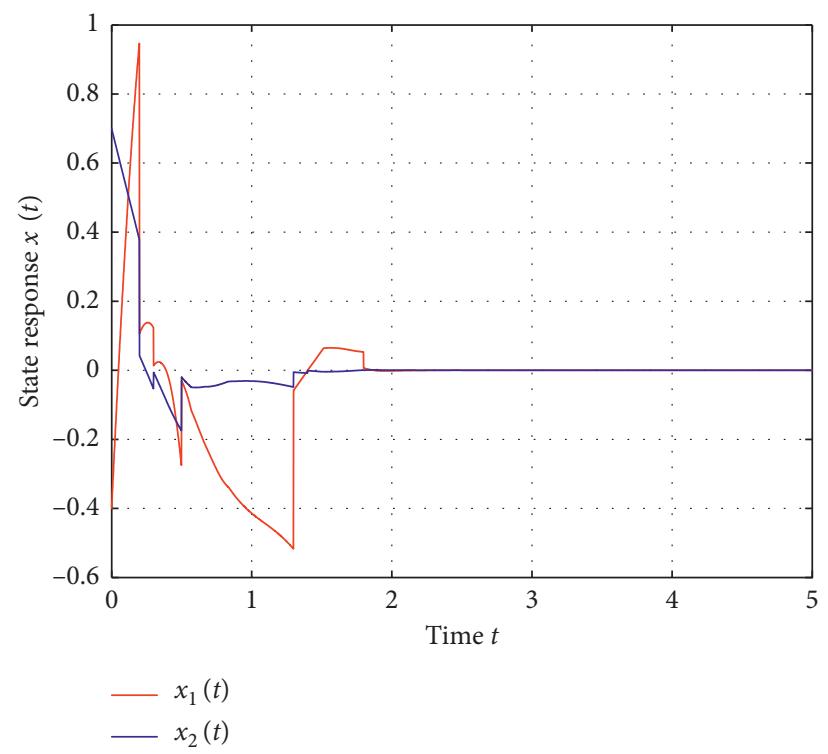

Figure 8: Curve of $x(t)$ with AEIC for Example 2. 


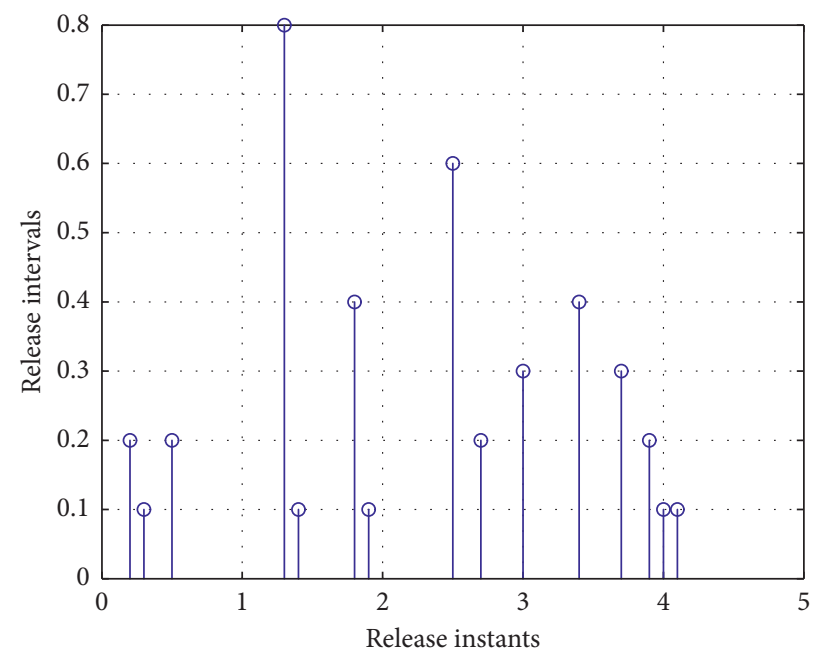

Figure 9: Release instants and intervals for Example 2.

$$
\begin{aligned}
& \Omega=10^{4} \times\left[\begin{array}{cc}
0.0424 & -0.0567 \\
-0.0567 & 1.4377
\end{array}\right], \\
& P=10^{3} \times\left[\begin{array}{cc}
0.0514 & -0.1625 \\
-0.1625 & 2.5712
\end{array}\right], \\
& W=\left[\begin{array}{cc}
24.3347 & -119.3639 \\
-119.3639 & 691.4778
\end{array}\right], \\
& R_{1}=10^{3} \times\left[\begin{array}{cc}
0.0154 & -0.1028 \\
-0.1028 & 1.0734
\end{array}\right], \\
& R_{2}=10^{3} \times\left[\begin{array}{cc}
0.0269 & -0.1600 \\
-0.1600 & 2.1631
\end{array}\right], \\
& R_{3}=10^{3} \times\left[\begin{array}{cc}
0.2830 & 0.4031 \\
0.4031 & 7.7438
\end{array}\right] .
\end{aligned}
$$

Let the neuron activation function $f_{i}(x)=\tanh (x)$, the time-varying delay $\delta(t)=0.2+0.2 \cos (t)$, the initial value $x(0)=[-0.4 ; 0.7]$, and the parameters $\Delta_{1}=1, \Delta_{2}=20$ in adaptive rule (6); Figures $7-10$ are presented to reflect intuitively the feasibility and validity of the obtained result in this paper. Figures 7 and 8 show the state response of systems (47) with parameters (56) and (57) under no any control and the well designed AEIC scheme (5), respectively. Clearly, AEIC scheme (5) is effective. Furthermore, it is not hard to see from Figure 9 that the frequency of impulsive control is reduced to a large extent, which means that more networks resources are saved by using the AEIC scheme. Moreover, compared with Figures 8 and 10, one can find that the triggering threshold adjusted adaptively by adaptive rule (6) is varying with the evolution of system state, i.e., the threshold gradually decreases when the norm of the system state goes up, which enhances the flexibility of the AEIC scheme to some extent.

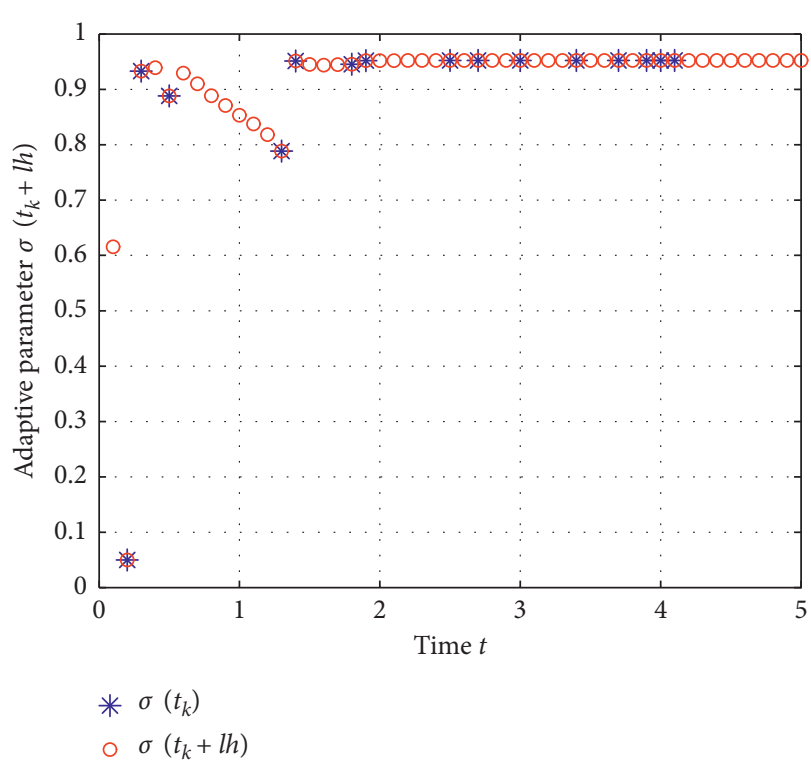

FIgURE 10: Adaptive parameters for Example 2.

\section{Conclusion}

In this paper, we have studied the stochastic exponential stabilization problem for MJNNs with TDs. A general LKF involving more information about sample data, Markov jump parameters, and TDs are constructed, and some exponential stabilization criteria for two kinds of NNs with TDs are established by employing a novel FMEII and a new AEIC scheme. The feasibility and validity of the obtained results are illustrated by two numerical examples. More importantly, the methods proposed in this paper can be employed to deal with other control issues for time delayed systems in our future work. 


\section{Data Availability}

The data used to support the findings of this study are included within the article.

\section{Conflicts of Interest}

The authors declare that there are no conflicts of interest regarding the publication of this paper.

\section{Acknowledgments}

This work was funded by the National Natural Science Foundation of China (Grant nos. 11671206 and 11601474) and Key Project of Natural Science Research of Anhui Higher Education Institutions of China (Grant no. KJ2018A0700).

\section{References}

[1] M. Galicki, H. Witte, J. Dörschel, M. Eiselt, and G. Griessbach, "Common optimization of adaptive preprocessing units and a neural network during the learning period. application in eeg pattern recognition," Neural Networks, vol. 10, no. 6, pp. 1153-1163, 1997.

[2] A. Rawat, R. N. Yadav, and S. C. Shrivastava, "Neural network applications in smart antenna arrays: a review," AEU - International Journal of Electronics and Communications, vol. 66, no. 11, pp. 903-912, 2012.

[3] S. Wen, T. Huang, Z. Zeng, Y. Chen, and P. Li, "Circuit design and exponential stabilization of memristive neural networks," Neural Networks, vol. 63, pp. 48-56, 2015.

[4] R. Saravanakumar, G. Rajchakit, M. S. Ali, and Y. H. Joo, "Exponential dissipativity criteria for generalized bam neural networks with variable delays," Neural Computing and Applications, vol. 31, no. 7, pp. 2717-2726, 2017.

[5] R. Saravanakumar, S. B. Stojanovic, D. D. Radosavljevic, C. K. Ahn, and H. R. Karimi, "Finite-time passivity-based stability criteria for delayed discrete-time neural networks via new weighted summation inequalities," IEEE Transactions on Neural Networks \& Learning Systems, vol. 30, no. 1, pp. 58-71, 2018.

[6] Z. Zhao, Q. Song, and S. He, "Passivity analysis of stochastic neural networks with time-varying delays and leakage delay," Neurocomputing, vol. 125, pp. 22-27, 2014.

[7] T. Jiao, G. Zong, S. K. Nguang, and C. Zhang, "Stability analysis of genetic regulatory networks with general random disturbances," IEEE Transactions on NanoBioscience, vol. 18, no. 2, pp. 128-135, 2019.

[8] Y. Zhang, Y. Yang, P. Shi, and J.-S. Pan, "Finite-time boundedness of markovain jump nonlinear systems with incomplete information," International Journal of Systems Science, vol. 49, no. 16, pp. 3296-3306, 2018.

[9] Z. Wang, Y. Liu, L. Yu, and X. Liu, "Exponential stability of delayed recurrent neural networks with markovian jumping parameters," Physics Letters A, vol. 356, no. 4-5, pp. 346-352, 2006.

[10] Y. Zhu, L. Zhang, and W. X. Zheng, "Distributed H filtering for a class of discrete-time markov jump lur'e systems with redundant channels," IEEE Transactions on Industrial Electronics, vol. 63, no. 3, pp. 1876-1885, 2016.

[11] J. Cheng, C. K. Ahn, H. R. Karimi, J. Cao, and W. Qi, “An event-based asynchronous approach to markov jump systems with hidden mode detections and missing measurements,"
IEEE Transactions on Systems, Man, and Cybernetics: Systems, vol. 49, no. 9, pp. 1749-1758, 2019.

[12] G. Wang, J. Cao, and J. Liang, "Exponential stability in the mean square for stochastic neural networks with mixed timedelays and markovian jumping parameters," Nonlinear Dynamics, vol. 57, no. 1-2, pp. 209-218, 2009.

[13] H. Shen, F. Li, Z.-G. Wu, J. H. Park, and V. Sreeram, "Fuzzymodel-based nonfragile control for nonlinear singularly perturbed systems with semi-markov jump parameters," IEEE Transactions on Fuzzy Systems, vol. 26, no. 6, pp. 3428-3439, 2018.

[14] Y. Zhang, P. Shi, and R. K. Agarwal, "Event-based dissipative analysis for discrete time-delay singular stochastic systems," International Journal of Robust and Nonlinear Control, vol. 28, no. 18, pp. 6106-6121, 2018.

[15] L. Tao, L. Qi, C. Sun, and B. Zhang, "Exponential stability of recurrent neural networks with time-varying discrete and distributed delays," Nonlinear Analysis Real World Applications, vol. 10, no. 4, pp. 2581-2589, 2009.

[16] L. Zhou, "Delay-dependent exponential stability of recurrent neural networks with markovian jumping parameters and proportional delays," Neural Computing and Applications, vol. 28, no. 1, pp. 765-773, 2017.

[17] Y. Liu, C. Zhang, Y. Kao, and C. Hou, "Exponential stability of neutral-type impulsive markovian jump neural networks with general incomplete transition rates," Neural Processing Letters, vol. 47, no. 2, pp. 325-345, 2018.

[18] X. Zhang, W. Zhou, and Y. Sun, "Exponential stability of neural networks with markovian switching parameters and general noise," International Journal of Control, Automation and Systems, vol. 17, no. 4, pp. 966-975, 2019.

[19] Y. He, M.-D. Ji, C.-K. Zhang, and M. Wu, "Global exponential stability of neural networks with time-varying delay based on free-matrix-based integral inequality," Neural Networks, vol. 77, pp. 80-86, 2016.

[20] R. Manivannan, G. Mahendrakumar, R. Samidurai, J. Cao, and A. Alsaedi, "Exponential stability and extended dissipativity criteria for generalized neural networks with interval time-varying delay signals," Journal of the Franklin Institute, vol. 354, no. 11, pp. 4353-4376, 2017.

[21] Y. Liu, J. H. Park, and F. Fang, "Global exponential stability of delayed neural networks based on a new integral inequality," IEEE Transactions on Systems, Man, and Cybernetics: Systems, vol. 49, no. 11, pp. 2318-2325, 2018.

[22] H. Trinh and L. Hien, "Exponential stability of time-delay systems via new weighted integral inequalities," Applied Mathematics and Computation, vol. 275, pp. 335-344, 2016.

[23] S. Vong, C. Shi, and D. Liu, "Improved exponential stability criteria of time-delay systems via weighted integral inequalities," Applied Mathematics Letters, vol. 86, pp. 14-21, 2018.

[24] C. Peng and F. Li, "A survey on recent advances in eventtriggered communication and control," Information Sciences, vol. 457-458, pp. 113-125, 2018.

[25] X. Tan, J. Cao, and X. Li, "Consensus of leader-following multiagent systems: a distributed event-triggered impulsive control strategy," IEEE Transactions on Cybernetics, vol. 49, no. 3, pp. 1-10, 2018.

[26] M. Cao, Z. Ai, and L. Peng, "Input-to-state stabilization of nonlinear systems via event-triggered impulsive control," IEEE Access, vol. 7, pp. 118581-118585, 2019.

[27] X. Li, H. Zhu, and S. Song, "Input-to-state stability of nonlinear systems using observer-based event-triggered impulsive control," IEEE Transactions on Systems, Man, and Cybernetics: Systems, 2020, In press). 
[28] Y. Zhou and Z. Zeng, "Event-triggered impulsive control on quasi-synchronization of memristive neural networks with time-varying delays," Neural Networks, vol. 110, pp. 55-65, 2019.

[29] M. S. Ali, R. Vadivel, and R. Saravanakumar, "Event-triggered state estimation for markovian jumping impulsive neural networks with interval time-varying delays," International Journal of Control, vol. 92, no. 2, pp. 270-290, 2019.

[30] W. Zhu, D. Wang, L. Liu, and G. Feng, "Event-based impulsive control of continuous-time dynamic systems and its application to synchronization of memristive neural networks," IEEE Transactions on Neural Networks and Learning Systems, vol. 29, no. 8, pp. 3599-3609, 2018.

[31] B. Liu, D. J. Hill, and Z. Sun, "Stabilisation to input-to-state stability for continuous-time dynamical systems via eventtriggered impulsive control with three levels of events," IET Control Theory \& Applications, vol. 12, no. 9, pp. 1167-1179, 2018.

[32] K. Gu, J. Chen, and V. L. Kharitonov, Stability of Time-Delay Systems, Springer Science \& Business Media, Berlin, Germany, 2003.

[33] P. Park, W. I. Lee, and S. Y. Lee, “Auxiliary function-based integral inequalities for quadratic functions and their applications to time-delay systems," Journal of the Franklin Institute, vol. 352, no. 4, pp. 1378-1396, 2015.

[34] R. Rakkiyappan, A. Chandrasekar, J. H. Park, and O. M. Kwon, "Exponential synchronization criteria for markovian jumping neural networks with time-varying delays and sampled-data control," Nonlinear Analysis: Hybrid Systems, vol. 14, pp. 16-37, 2014.

[35] A. Chandrasekar, R. Rakkiyappan, F. A. Rihan, and S. Lakshmanan, "Exponential synchronization of markovian jumping neural networks with partly unknown transition probabilities via stochastic sampled-data control," Neurocomputing, vol. 133, no. 10, pp. 385-398, 2014.

[36] H. Zhang, J. Wang, Z. Wang, and H. Liang, "Sampled-data synchronization analysis of markovian neural networks with generally incomplete transition rates," IEEE Transactions on Neural Networks and Learning Systems, vol. 28, no. 3, pp. 740-752, 2017. 